\title{
LDA Feature Selection for Satellite Image Fusion in HAAR Wavelet
}

\author{
Anfal Hazim Abdullah ${ }^{1}$, Dr. E. Sreenivasa Reddy ${ }^{2}$ \\ ${ }^{1}$ PG Scholar, Dept. of CSE, University College of Engineering \& Technology, Acharya Nagarjuna University, Guntur, 522510, Andhra \\ Pradesh, India \\ ${ }^{2}$ Professor, Dean and HOD of CSE, University College of Engineering \& Technology, Acharya Nagarjuna University, Guntur, 522510, \\ Andhra Pradesh, India
}

\begin{abstract}
Image fusion is a technique that integrate complimentary details from multiple input images such that the new image give more information and more suitable for the purpose of human visual perception. Major technical constraints like minimum data storage at satellite platform in space, less bandwidth for communication with earth station, etc. limits the satellite sensors from capturing images with high spatial and high spectral resolutions simultaneously. To overcome this limitation, image fusion has proved to be a potential tool in remote sensing applications which integrates the information from combinations of panchromatic, multispectral or hyperspectral images; intended to result in a composite image having both higher spatial and higher spectral resolutions. This paper presents new algorithm to fuse the multi-image in four steps. First discrete wavelet transform (DWT) for time to frequency conversion, feature extraction, feature selection based on LDA and finally classify the feature level fusion. Here LDA will select best feature of the multiple image to fuse the image.
\end{abstract}

Keywords: Image fusion, Relative spectral contribution methods, Component substitution, Multi-resolution analysis, Quality metrics for performance evaluation, LDA feature selection

\section{Introduction}

A wide variety of remotely sensed data products like panchromatic (PAN), multispectral (MS), hyperspectral (HS), synthetic aperture radar (SAR) imagery, etc. covering different parts of electromagnetic spectrum are made available by different earth observation satellites. These remote sensing data products are further processed and used for crop production forecast, forest cover and type mapping, mineral/oil exploration, weather prediction, watershed development and monitoring, urban sprawl mapping of major cities, disaster management, etc. are the flow of tasks in remote sensing image processing[1][2]. For many of the applications listed, image analysis of only one source type is insufficient. For detail understanding of the observed earth surface, it is better to get complementary information from more than one sensor. Thus, image fusion becomes the best option to integrate information collected from different imaging sensors at different spectral, spatial, temporal and radiometric resolutions. Image fusion is a subset of more diverse research area data fusion' which is defined as: Đata fusion is a formal framework in which are expressed means and tools for the alliance of data originating from different sources. It aims at obtaining information of greater quality; the exact definition of greater quality ${ }^{\star}$ will depend upon the application"[3]. Image fusion takes place at three different levels.

Sparse representation of signals is now possible utilizing many different Greedy approaches including Matching Pursuit, Orthogonal Matching Pursuit. These techniques are used to represent signals with the fewest number of non-zero coefficients. Principal Component Analysis is one of the powerful state-of-theartimage fusion approaches in terms of visual inspection and quantitative evaluation metrics. This fusion is carried out by integrating the principal components of images to be fused. PCA is one of the special domain fusion approach[4]. DWT is one of the transform domain fusion approach. In DWT cost of computing is high and takes long compression time. Both PCA and Sparse fusion have specific advantages and disadvantages. PCA fusion will enhance the spatial quality but have dense nonzero entries that might represent uninformative features[3][5]. Sparse fusion preserves important information but high spatial resolution is lacking. An algorithm which utilizes the advantages of both PCA and Sparse representation for fusing common and innovative features of the captured images is proposed. This algorithm also overcomes the disadvantages of both PCA and Sparse representation. The effectiveness of proposed method by comparing its results with PCA and Sparse Fusion is demonstrated [5].

\subsection{Pixel Level Image Fusion}

Pixel-level image fusion is the lowest level of image fusion, where a new image is formed having pixel values obtained by combining the pixel values of different images through some algorithms under strict registration conditions. The new image keeps more raw data to provide rich and accurate image information which is further used for easy analysis and processing by feature extraction and classification. The image fusion at pixel level may be single sensor, multisensor or temporal image fusion, etc. Advantage of pixellevel image fusion is minimum loss of information, but it has the largest amount of information to be processed, thus slowest processing speed, and a higher demand for equipment [1][2].

\subsection{Feature Level Image Fusion}

Feature-level fusion is intermediate level of image fusion where the features (edges, texture, shape, spectrum, angle or direction, speed, similar lighting area, similar depth of focus area, etc.) generally in statics are extracted from different images of the same geographical area by separate preprocessing. The extracted features are combined to form 


\section{International Journal of Science and Research (IJSR) \\ ISSN (Online): 2319-7064 \\ Index Copernicus Value (2013): 6.14 | Impact Factor (2015): 6.391}

an optimum feature set, further classified using statistical or other types of classifiers. Features from different sourceimages preprocessed using different schemes are combined to form a decision. Here, correlative feature information is excavated, redundant features are eliminated and new compound features are established, increasing the degree of reliability of feature information. The requirement of sensor alignment in feature-level fusion is less strict than pixellevel's. So, image sensors can be distributed in different platforms. The advantages of feature-level fusion are that it achieved considerable compression of information, it is conducive to real-time processing and its fusion results can further give the feature information for decision analysis. Here, the extracted features are directly related to the decision analysis[1][2].

\subsection{Decision Level Image Fusion}

Decision-level fusion is a high-level fusion, and its results provide the basis for command and control decision making. In decision-level fusion, the images are processed separately. The processed information is then refined by combining the information obtained from different sources and the differences in information are resolved based on certain decision rules. In literature, two types of decision level fusion are observed. Here, classification from different types of classifiers for the same image may be combined to get better classification accuracies or two different complimentary sources like optical imagery and radar data can be classified separately and combined to produce a refined classification map. A variety of logical reasoning methods, statistical methods, information theory methods can be used for decision-level fusion, such as Bayesian reasoning, D-S (Dempster-Shafer) evidence reasoning, voting system, cluster analysis, fuzzy set theory, neural network , the entropy method and so on. Decision-level fusion has a good real-time and fault tolerance, but its pretreatment cost is higher. The data quantity of decisionlevel fusion is the smallest and its ability of anti-interference is the highest. The probability and reality of fused results are high and the performance of multi-sensor system is improved[1][2].

The remainder of the paper is organized as follows: Section II presents a brief review of some of the image fusion approaches published earlier. Section III is devoted to image fusion methods belonging to different approaches, providing a detailed description of some algorithms and dwt. Section IV describes the feature extraction and LDA feature selection. Section V quality metrics for the evaluation of image fusion performance based on two assessment procedures operating at reduced and full resolutions. Future scope and limitations of the research are discussed in Section V. Finally, conclusions are drawn in Section VI.

\section{Literature Review}

To know the current state of art of image fusion in remote sensing, this section reviews some of the research papers published earlier. For fusion of MS image with PAN image (also called as pansharpening), it is observed that the effective high quality is achieved with the expense of large computational complexity, more time consumption and difficulties to process large size images. Along with this, Gemine Vivone et al. in focused some important points as: the lack of universally recognized evaluation criteria, unavailability of image data sets for benchmarking and absence of standardized implementations of the algorithms to make a thorough evaluation and comparison of the different pansharpening methods. Pansharpening algorithms belonging to the two more established and addressed categories viz. component substitution (CS) and multiresolution analysis (MRA) are considered by authors to be evaluated and compared over fivedata sets acquired by different satellites[4]. Wenqing Wang et al. Further propose an adaptive component substitution based pansharpening, adopting a particle-swarm-optimization algorithm to solve the single objection optimization problem. The proposed framework is compared with popular CS based methods based on correlation coefficient, mutual information and mean-structural-similarity index. Andrea Garzelli et al. while extending this classical component-substitution approach, propose to develop a fast nonlocal parameter based pansharpening method K-means clustering and overcoming window-based local estimation. In a try to solve the problem of color distortion, Qizhi Xu et al. divides the pixels of PAN and MS images into several classes by $k$ means algorithm followed by multiple regression to calculate summation weights on each group of pixels. It is also noticed that due to the nonlinear spectral response of satellite sensors, synthesis of low-resolution PAN image required in some methods cannot be well approximated resulting in color distortion[6].

Other than fusion of MS with PAN image, fusion of MS with HS images is also seen in literature. Due to complexity and cost issues, MS and HS images have different resolutions with significantly lower spatial resolution than that of PAN images. HS remote sensing images have advantage of more information content due to large number of bands involved, but the same time its application has been constrained due to the narrow swath width. Thus, a fusion of HS and MS images is proposed many times. Miguel Simões et al. formulate this data fusion problem as the minimization of blur, additive noise, etc. promoting piecewise-smooth solutions. Author estimate a hard estimation problem in accounting different spatial resolutions, handling very large size of HS image together, etc. which is performed by following an Alternating Direction Method of Multipliers (ADMM) approach and using the Split Augmented Lagrangian Shrinkage Algorithm (SALSA). Here, a blind approach focusing the inherent data redundancy to achieve better results is followed. Xuejian Sun et al. proposes a spectral resolution enhancement method (SREM) for remotely sensed MS image, to generate wide swath HS images using auxiliary multi/hyper-spectral data[7]. Transformation matrices are generated followed by a spectral angle weighted minimum distance (SAWMD) matching method to create HS vectors from the original MS image, pixel by pixel. Further Caroline M. Gevaert et al. performs fusion of MS and HS images acquired with Formosat- 2 and an unmanned aerial vehicle (UAV) respectively to construct Spectral-temporal response surfaces (STRSs) providing continuous reflectance spectra at high temporal intervals for precision agriculture requirements. The method uses Bayesian theory to impute 


\section{International Journal of Science and Research (IJSR) \\ ISSN (Online): 2319-7064 \\ Index Copernicus Value (2013): 6.14 | Impact Factor (2015): 6.391}

missing spectral information in the MS imagery. The performance of method is evaluated on field measured reflectance spectra, leaf area index (LAI), etc. On the other hand, Qi Wei et al. present an approach for fusing HS and MS images based on sparse representation. An inverse problem of image fusion is formulated, assuming the target image to live in a lower dimensional subspace. Dictionary learning with design of a sparse regularization term are done and results are simulated for comparison to preexisting methods. To enhance spatial resolution of MS and HS images, Frosti Palsson et al. proposes a method for fusion of MS or HS with PAN images and MS with HS images. Spectral redundancy is tried to be reduced by principal component analysis (PCA) and use of wavelets. The approach is said to have benefits of substantially lower computational requirements and very high tolerance to noise for WorldView-2 data[3][4].

Image fusion has wide application area. Claudia Paris et al. based on the fusion between low-density LiDAR (Light detection and ranging) data and high-resolution optical images proposes a 3-D model-based approach to the estimate tree top height as one of the forest attributes. Difficulties and ambiguities in object recognition of urban areas are tried to be avoided by Fatemeh Tabib Mahmoudi et al. The authors propose object-based image analysis (OBIA) to be performed in case of single view processes; while in multi-views' case, all the views are to be fused at decision level. The approach is implemented to produce results for WorldView-2 and the digital modular camera (DMC) datasets. Network transmissions for surveillance in radar systems are generally narrowband. Gianluca Gennarelli et al. propose to use single-frequency approach for imaging targets with passive arrays deployed around the scattering scene. Employing a multi-array image fusion strategy in conjunction with a change detection scheme for imaging moving targets, better resolution images are obtained. The author carries some numerical estimation using this approach. Yong Xu et al develop a spatial and temporal data fusion model based on regularized spatial unmixing to generate Landsat-like synthetic data and the high temporal resolution of Moderate Resolution Imaging Spectro-radiometer (MODIS) data. After considering the neighborhood size of the MODIS data, the number of classes of Landsat data for spatial unmixing and a regularization parameter added to the cost function to reduce unmixing error the approach is evaluated by calculating ERGAS. Wei Li et al. propose a classification paradigm to exploit rich texture information of HS image. The framework employs local binary patterns (LBPs) to extract features such as edges, corners and spots. Feature-level fusion and decisionlevel fusion are applied to the extracted LBP features before classification.

\section{Image Fusion Methods}

\subsection{Pixel Level Image Fusion}

Pixel-level image fusion is the lowest level of image fusion, where a new image is formed having pixel values obtained by combining the pixel values of different images through some algorithms under strict registration conditions. The new image keeps more raw data to provide rich and accurate image information which is further used for easy analysis and processing by feature extraction and classification. The image fusion at pixel level may be single sensor, multisensor or temporal image fusion, etc. Advantage of pixellevel image fusion is minimum loss of information, but it has the largest amount of information to be processed, thus slowest processing speed, and a higher demand for equipment [11].

\subsection{Average}

It is a well-documented fact that regions of images that are in focus tend to be of higher pixel intensity. Thus, this algorithm is a simple way of obtaining an output image with all regions in focus. In this method the resultant fused image is obtained by taking the average intensity of corresponding pixels from both the input image [11].

$$
F_{i j}=\frac{\left(A_{i j}+B_{i j}\right)}{2}
$$

\subsection{Select Maximum}

The greater the pixel values the more in focus the image. Thus this algorithm chooses the in-focus regions from each input image by choosing the greatest value for each pixel, resulting in highly focused output. In this method, the resultant fused image is obtained by selecting the maximum intensity of corresponding pixels from both the input images [13].

$$
F_{i j}=\sum_{i=0}^{m} \sum_{j=0}^{n} \max A_{i j} B_{i j}
$$

\subsection{Select Minimum}

In this method, the resultant fused image is obtained by selecting the minimum intensity of corresponding pixels from both the input images [13].

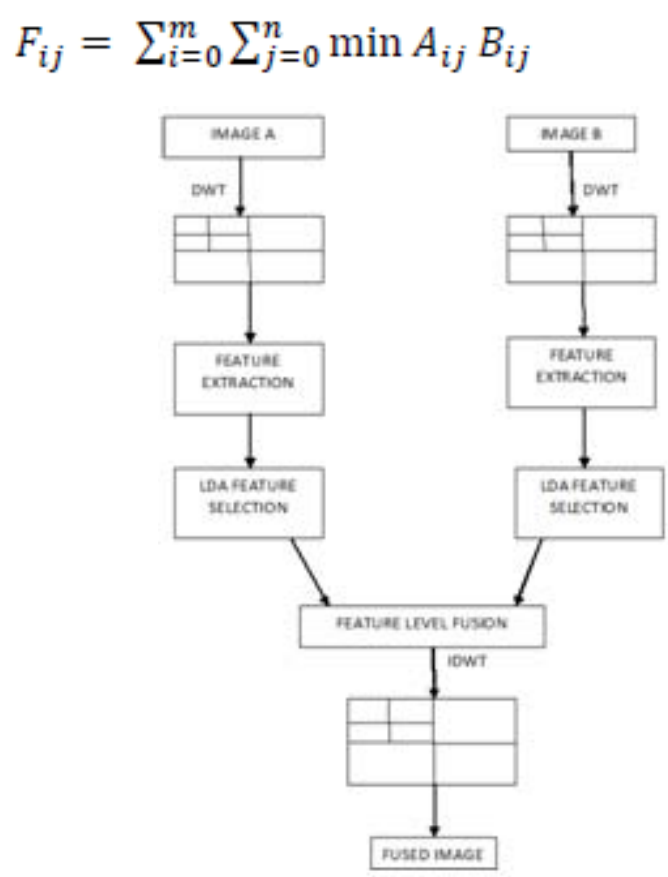

Figure 1: Block Diagram 


\section{International Journal of Science and Research (IJSR) \\ ISSN (Online): 2319-7064}

Index Copernicus Value (2013): 6.14 | Impact Factor (2015): 6.391

\subsection{Feature Level Image Fusion}

Feature-level fusion is intermediate level of image fusion where the features (edges, texture, shape, spectrum, angle or direction, speed, similar lighting area, similar depth of focus area, etc.) generally in statics are extracted from different images of the same geographical area by separate preprocessing [6]. The extracted features are combined to form an optimum feature set, further classified using statistical or other types of classifiers. Features from different source-images preprocessed using different schemes are combined to form a decision.

Here, correlative feature information is excavated, redundant features are eliminated and new compound features are established, increasing the degree of reliability of feature information. The requirement of sensor alignment in featurelevel fusion is less strict than pixel-level's. So, image sensors can be distributed in different platforms. The advantages of feature-level fusion are that it achieved considerable compression of information, it is conducive to real-time processing and its fusion results can further give the feature information for decision analysis. Here, the extracted features are directly related to the decision analysis [7].

\subsection{Intensity-Hue-Saturation (IHS)}

The intensity-hue-saturation (IHS) method is a standard procedure in the area of image fusion having fast computing capability for fusing images. Digital images in computer are generally displayed by an additive color composite system using the three primary colors - red (R), green $(G)$ and blue (B). Since, perception of colors to humans always recognize with three features - intensity $(\mathrm{I})$, hue $(\mathrm{H})$ and saturation $(\mathrm{S})$, the IHS fusion method converts a color multispectral (MS) image from the RGB space into the IHS color space [34]. While the RGB is a rectangular coordinate system and IHS is a cylindrical coordinate system, the conversion is performed by rotation of axis from the orthogonal RGB system to a new orthogonal IHS system [15]. The intensity represents the overall brightness or luminance of the scene of any color, the hue component refers to dominant wavelength of the light contributing to the scene and saturation component describes its purity. Most literature recognizes IHS as a third-order method because it employs a $3 \times 3$ matrix in its transform kernel. The appearance of the panchromatic (PAN) image is similar to the intensity band of an IHS representation of the scene. Therefore, during fusion i.e. pan-sharpening, the MS image is projected onto the IHS color space and the intensity band is replaced by the PAN image. The fusion output can then be obtained by the reverse IHS transform [36]. The steps in IHS method are given below, where the equations (10), (11), (12) give mathematical interpretations of forward transformation while equation (13) gives IHS reverse transformation.

1) The low resolution MS imagery is co-registered to the same area as the high resolution PAN imagery and resampled to the same resolution as the PAN imagery. It is generally performed using bi-cubic interpolation.
2) The three resampled bands of the MS imagery, which represent the RGB space are transformed into IHS components. It is mathematically represented as follows-

$$
\begin{aligned}
& {\left[\begin{array}{c}
I \\
V_{1} \\
V_{2}
\end{array}\right]=\left[\begin{array}{ccc}
\frac{1}{3} & \frac{1}{3} & \frac{1}{3} \\
\frac{1}{\sqrt{6}} & \frac{1}{\sqrt{6}} & \frac{-2}{\sqrt{6}} \\
\frac{1}{\sqrt{2}} & \frac{-1}{\sqrt{2}} & 0
\end{array}\right]\left[\begin{array}{l}
R \\
G \\
B
\end{array}\right]} \\
& H=\tan ^{-1}\left(\frac{V_{2}}{V_{1}}\right) \\
& S=\sqrt{V_{1}^{2}+V_{2}^{2}}
\end{aligned}
$$

Where, V1 and V2 are intermediate variables.

3. The PAN imagery is histogram matched to the $\underline{\underline{I}}^{\text {c }}$ component. This is done in order to compensate for the spectral differences between the two images, which occurred due to different sensors or different acquisition dates and angles.

4. The intensity component of MS imagery is replaced by the histogram matched PAN imagery. The RGB of the new merged MS imagery is obtained by computing a reverse IHS to RGB transform. Following equation gives IHS inverse transform.

$$
\left[\begin{array}{l}
R \\
G \\
B
\end{array}\right]=\left[\begin{array}{ccc}
1 & \frac{1}{\sqrt{6}} & \frac{1}{\sqrt{2}} \\
1 & \frac{1}{\sqrt{6}} & \frac{-1}{2} \\
\frac{11}{\sqrt{2}} & \frac{-2}{\sqrt{6}} & 0
\end{array}\right]\left[\begin{array}{c}
I \\
V_{1} \\
V_{2}
\end{array}\right]
$$

\subsection{Decision Level Image Fusion}

Decision-level fusion is a high-level fusion, and its results provide the basis for command and control decision making. In decision-level fusion, the images are processed separately. The processed information is then refined by combining the information obtained from different sources and the differences in information are resolved based on certain decision rules. In literature, two types of decision level fusion are observed. Here, classification from different types of classifiers for the same image may be combined to get better classification accuracies or two different complimentary sources like optical imagery and radar data can be classified separately and combined to produce a refined classification map. A variety of logical reasoning methods, statistical methods, information theory methods can be used for decision-level fusion, such as Bayesian reasoning, D-S (Dempster-Shafer) evidence reasoning, voting system, cluster analysis, fuzzy set theory, neural network , the entropy method and so on. Decision-level fusion has a good real-time and fault tolerance, but its pretreatment cost is higher. The data quantity of decisionlevel fusion is the smallest and its ability of anti-interference is the highest. The probability and reality of fused results are 


\section{International Journal of Science and Research (IJSR) \\ ISSN (Online): 2319-7064 \\ Index Copernicus Value (2013): 6.14 | Impact Factor (2015): 6.391}

high and the performance of multi-sensor system is improved. Accompanied image fusion schemes at three different levels[3][5].

\subsection{Wavelet Transform based Methods:}

A „wavelet' as its name implies is a small wave that grows and decays essentially in a limited time period. Its use as wavelet transform is an extension of Fourier transform in many aspects and has been successfully used in image fusion. The signal here is projected on a set of wavelet functions to get best resolution without altering the spectral contents of the image. This multi-resolution approach is suited to different resolutions, which allows the image decomposition in different kinds of coefficients. The coefficients from different images are combined to form new coefficients, which after inverse transformation gives fused image [15]. It provides good resolution in both time and frequency domains. It is also found to have advantages over pyramid methods in terms of increased directional information, no blocking artifacts, better signal to noise ratios and improved perception

A function can be called a wavelet if it satisfies two basic properties:

Its time integral must be zero i.e.

$$
\int_{-\infty}^{\infty} \psi(t) d t=0
$$

and square of wavelet integrated over time is unity.

$$
\int_{-\infty}^{\infty} \psi^{2}(t) d t=1
$$

\section{A family of wavelets can be generated by dilating and translating the mother wavelet which is given by}

$$
\psi_{a, b}(x)=\frac{1}{\sqrt{a}} \psi\left(\frac{x-b}{a}\right)
$$

Here, $a$ is the scale parameter and $b$ is the shift parameter.

\section{Feature Extraction}

In machine learning, pattern recognition and in image processing, feature extraction starts from an initial set of measured data and builds derived values (features) intended to be informative and non-redundant, facilitating the subsequent learning and generalization steps, and in some cases leading to better human interpretations. Feature extraction is related to dimensionality reduction.

When the input data to an algorithm is too large to be processed and it is suspected to be redundant (e.g. the same measurement in both feet and meters, or the repetitiveness of images presented as pixels), then it can be transformed into a reduced set of features (also named a features vector). This process is called feature extraction. The extracted features are expected to contain the relevant information from the input data, so that the desired task can be performed by using this reduced representation instead of the complete initial data.
One very important area of application is image processing, in which algorithms are used to detect and isolate various desired portions or shapes (features) of a digitized image or video stream. It is particularly important in the area of optical character recognition.

Low-level

- Edge detection

- Corner detection

- Blob detection

- Ridge detection

\subsection{LDA Feature Selection}

In practice, LDA for dimensionality reduction would be just another preprocessing step for a typical machine learning or pattern classification task.

\section{Step 1: Computing the d-dimensional mean vectors:}

In this first step, we will start off with a simple computation of the mean vectors $\mathbf{m}_{\mathbf{i}}$, $(i=1,2,3)$ of the 3 different flower classes:

$$
\boldsymbol{m}_{i}=\left[\begin{array}{c}
\mu_{\omega_{i} \text { (sepal length) }} \\
\mu_{\omega_{i} \text { (sepal width) }} \\
\mu_{\omega_{i} \text { (petal length) }} \\
\mu_{\omega_{i} \text { (petal width) }}
\end{array}\right], \text { with } i=1,2,3
$$

Step 2: Computing the Scatter Matrices:

Now, we will compute the two $4 x 4$-dimensional matrices: The within-class and the between-class scatter matrix.

\subsection{Within-class scatter matrix $S_{W}$}

The within-class scatter matrix $\mathbf{S}_{\mathbf{W}}$ is computed by the following equation:

$$
S_{W}=\sum_{i=1}^{c} S_{i}
$$

Where

$$
S_{i}=\sum_{\boldsymbol{x} \in D_{i}}^{n}\left(\boldsymbol{x}-\boldsymbol{m}_{i}\right)\left(\boldsymbol{x}-\boldsymbol{m}_{i}\right)^{T}
$$

(Scatter matrix for every class)

and $\mathbf{m}_{\mathbf{i}}$ is the mean vector

$$
\boldsymbol{m}_{i}=\frac{1}{n_{i}} \sum_{\boldsymbol{x} \in D_{i}}^{n} \boldsymbol{x}_{k}
$$

Alternatively, we could also compute the class-covariance matrices by adding the scaling factor "1 / (N-1)" to the within-class scatter matrix, so that our equation becomes

$$
\begin{aligned}
& \Sigma_{i}=\frac{1}{N_{i}-1} \sum_{\boldsymbol{x} \in D_{i}}^{n}\left(\boldsymbol{x}-\boldsymbol{m}_{i}\right)\left(\boldsymbol{x}-\boldsymbol{m}_{i}\right)^{T} . \\
& \text { and } S_{W}=\sum_{i=1}^{c}\left(N_{i}-1\right) \Sigma_{i}
\end{aligned}
$$




\section{International Journal of Science and Research (IJSR) \\ ISSN (Online): 2319-7064 \\ Index Copernicus Value (2013): 6.14 | Impact Factor (2015): 6.391}

Where $N_{i}$ is the sample size of the respective class (here: $50)$, and in this particular case, we can drop the term $\left(N_{i}-1\right)$ since all classes have the same sample size.

However, the resulting Eigenspaces will be identical (identical eigenvectors, only the eigenvalues are scaled differently by a constant factor).

The between-class scatter matrix $\mathbf{S}_{\mathbf{B}}$ is computed by the following equation:

$$
S_{B}=\sum_{i=1}^{c} N_{i}\left(\boldsymbol{m}_{i}-\boldsymbol{m}\right)\left(\boldsymbol{m}_{i}-\boldsymbol{m}\right)^{T}
$$

Wherem is the overall mean, and $\mathbf{m}_{\mathbf{i}}$ and $\mathbf{N}_{\mathbf{i}}$ are the sample mean and sizes of the respective classes.

Step 3: Solving the generalized eigenvalue problem for the matrix $S_{W^{-1}} S_{B}$ :

Next, we will solve the generalized eigenvalue problem for the matrix $\mathbf{S}_{\mathbf{W}}{ }^{-1} \mathbf{S}_{\mathbf{B}}$ to obtain the linear discriminants.

After this decomposition of our square matrix into eigenvectors and eigenvalues, let us briefly recapitulate how we can interpret those results. As we remember from our first linear algebra class in high school or college, both eigenvectors and eigenvalues are providing us with information about the distortion of a linear transformation: The eigenvectors are basically the direction of this distortion, and the eigenvalues are the scaling factor for the eigenvectors that describing the magnitude of the distortion.

If we are performing the LDA for dimensionality reduction, the eigenvectors are important since they will form the new axes of our new feature subspace; the associated eigenvalues are of particular interest since they will tell us how "informative" the new "axes" are.

Let us briefly double-check our calculation and talk more about the eigenvalues in the next section.

Checking the eigenvector-eigenvalue calculation:

A quick check that the eigenvector-eigenvalue calculation is correct and satisfy the equation:

$$
\boldsymbol{A} \boldsymbol{v}=\lambda \boldsymbol{v}
$$

$$
\begin{aligned}
& \text { where } \\
& \boldsymbol{A}=S_{W}^{-1} S_{B} \\
& \boldsymbol{v}=\text { Eigenvector } \\
& \lambda=\text { Eigenvalue }
\end{aligned}
$$

Step 4: Selecting linear discriminants for the new feature subspace

\subsection{Sorting the eigenvectors by decreasing eigenvalues}

Remember from the introduction that we are not only interested in merely projecting the data into a subspace that improves the class reparability, but also reduces the dimensionality of our feature space, (where the eigenvectors will form the axes of this new feature subspace).
However, the eigenvectors only define the directions of the new axis, since they have all the same unit length 1 .

So, in order to decide which eigenvector(s) we want to drop for our lower-dimensional subspace, we have to take a look at the corresponding eigenvalues of the eigenvectors. Roughly speaking, the eigenvectors with the lowest eigenvalues bear the least information about the distribution of the data, and those are the ones we want to drop. The common approach is to rank the eigenvectors from highest to lowest corresponding eigenvalue and choose the top $k$ eigenvectors.

\subsection{Choosing $k$ eigenvectors with the largest eigenvalues:}

After sorting the eigenpairs by decreasing eigenvalues, it is now time to construct our $d \times k$-dimensional eigenvector matrix W (here: $4 \times 2$ : based on the 2 most informative eigenpairs) and thereby reducing the initial 4-dimensional feature space into a 2-dimensional feature subspace.

Step 5: Transforming the samples onto the new subspace:

In the last step, we use the $4 \times 2$-dimensional matrix $W$ that we just computed to transform our samples onto the new subspace via the equation $\boldsymbol{Y}=\boldsymbol{X} \times \boldsymbol{W}$ (where $\boldsymbol{X}$ is an $n \times d$ dimensional matrix; the ith row represents the ith sample, and $\mathbf{Y}$ is the transformed $n \times k$-dimensional matrix with the $n$ samples projected into the new subspace).

\section{Experimental Results}

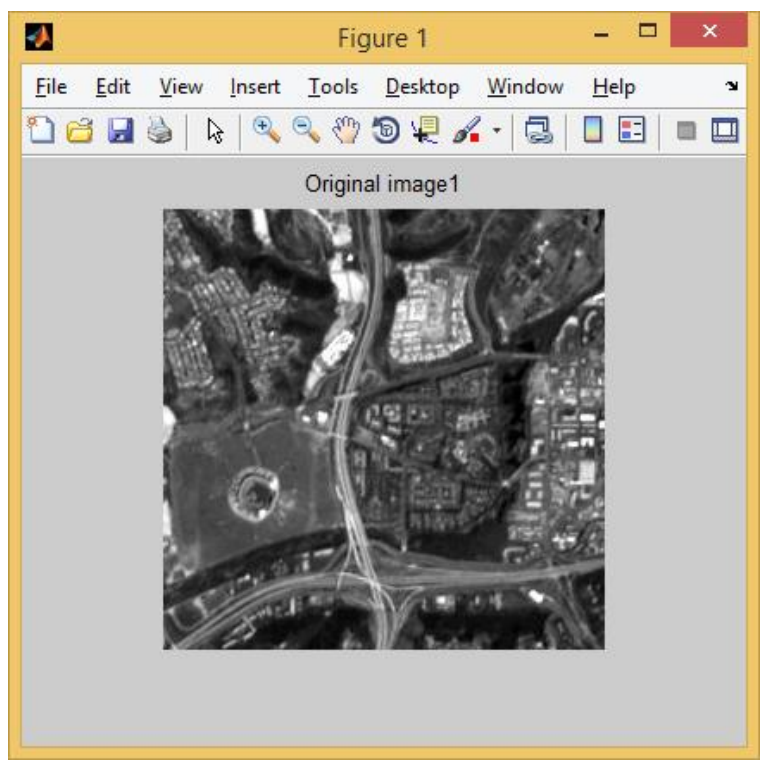

Figure 2(a): Original image1 


\section{International Journal of Science and Research (IJSR) \\ ISSN (Online): 2319-7064}

Index Copernicus Value (2013): 6.14 | Impact Factor (2015): 6.391

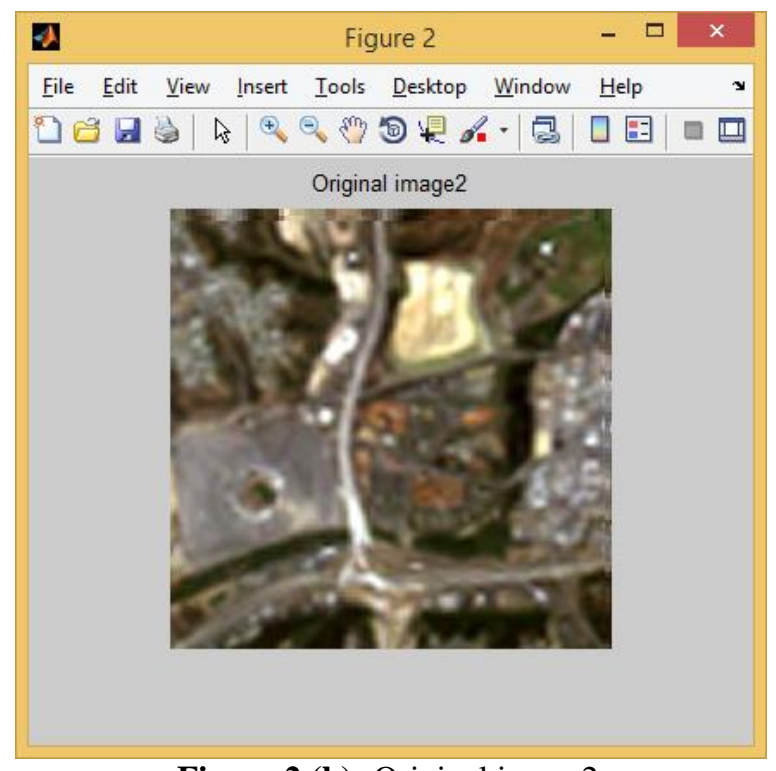

Figure 2 (b): Original image2

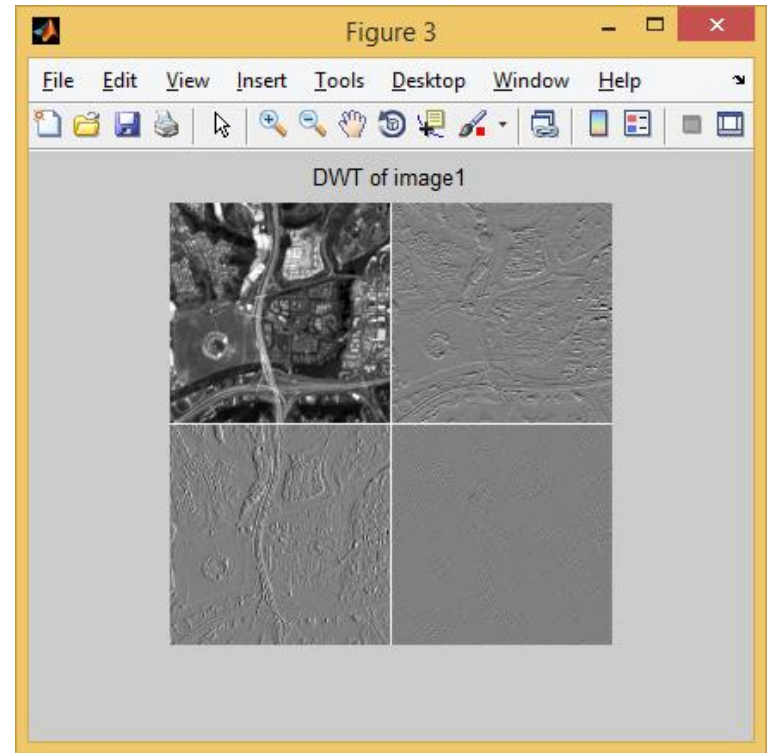

Figure 3(a): Discrete Wavelet Transform of image1

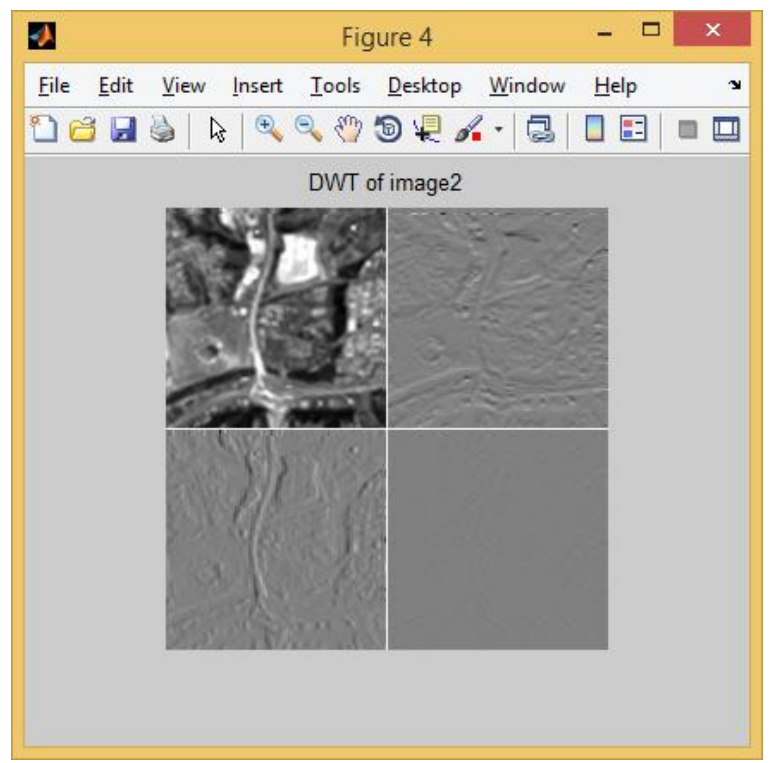

Figure 3 (b): Discrete Wavelet Transform of image2

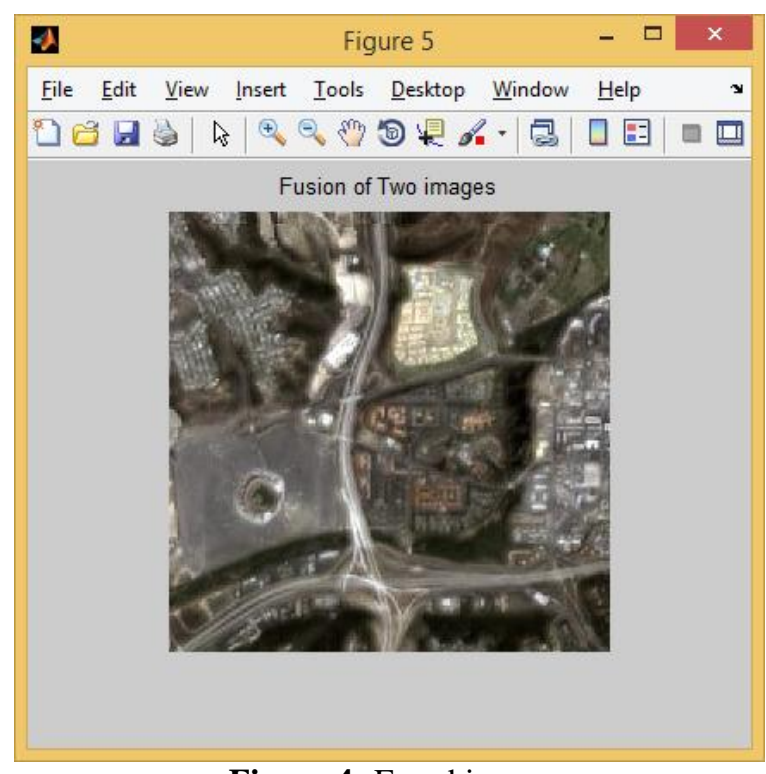

Figure 4: Fused image

Here the process of fusion is showed in the Fig.

\section{Performance Analysis}

\subsection{Standard Deviation $(\sigma)$}

Standard deviation is more efficient in the absence of noise. It measures the contrast in the fused image [6] [34]. An image with high contrast would have a high standard deviation given by -

$$
\sigma=\sqrt{\sum_{i=o}^{L}\left(i-i^{\prime}\right)^{2} h_{I f}(i), \ldots i^{\prime}}=\sum_{i=0}^{L} i h_{I f}
$$

Where, $h_{I f}(i)$ is the normalized histogram of the fused image $\operatorname{If}(\mathrm{x}, \mathrm{y})$ and $\mathrm{L}$ is the number of frequency bins in histogram.

\subsection{Mean Square Error (MSE)}

The mathematical equation of MSE is given by -

$$
M S E=\frac{1}{m n} \sum_{i=1}^{m} \sum_{j=1}^{n}\left(A_{i j}-B_{i j}\right)^{2}
$$

Where, $\mathrm{A}$ is the reference image, $\mathrm{B}$ is the fused image to be assessed, $i$ is the pixel row index,j is the pixel column index, $\mathrm{m}$ is number of rows and $\mathrm{n}$ is number of columns.

\subsection{Root Mean Square Error (RMSE)}

The RMSE is another standard measure of difference between the reference image and the fused image given by

$$
R M S E=\left(\frac{\sum_{i=1}^{M} \sum_{j=1}^{N}\left[I_{R}(i, j)-I_{F}(i, j)\right]^{2}}{M \times N}\right)^{\frac{1}{2}}
$$

Where, $I_{R}(i, j)$ and $I_{F}(i, j)$ are the image pixel values of the reference image and the fused image respectively. M X $\mathrm{N}$ is the image size. Greater the RMSE, higher the difference between reference and fused image will be. The main drawback of RMSE is that errors in each band are not related to the mean value of the band itself. 


\section{International Journal of Science and Research (IJSR) \\ ISSN (Online): 2319-7064}

Index Copernicus Value (2013): 6.14 | Impact Factor (2015): 6.391

\subsection{Peak to Peak Signal to Noise Ratio (PSNR)}

PSNR is the ratio between the maximum possible power of a signal and the power of corrupting noise that affects the fidelity of its representation. The PSNR measure is given by

$$
\operatorname{PSNR}(d B)=20 \log \frac{255 \sqrt{3 M N}}{\sqrt{\sum_{i=1}^{M} \sum_{j=1}^{N}\left(B^{\prime}(i, j)-B(i, j)\right)^{2}}}
$$

Where, $\mathrm{B}$ is the reference image, $\mathrm{B}$ ' is the fused image to be assessed, $\mathrm{i}$ is the pixel row index, $\mathrm{j}$ is the pixel column index, $\mathrm{M}$ is number of rows and $\mathrm{N}$ is the number of columns.

Table 1

\begin{tabular}{|c|c|c|c|c|}
\hline S/No & STD & RMSE & MSE & PSNR \\
\hline Image Set1 & 45.5546 & 6.5713 & 449.3667 & 43.2089 \\
\hline Image Set2 & 52.7232 & 3.9967 & 137.7656 & 53.4785 \\
\hline Image Set3 & 49.1491 & 3.9232 & 56.3901 & 59.5955 \\
\hline
\end{tabular}

Table 1: Shows the performance analysis of the proposed method.

\section{Conclusion}

Thus our proposed new algorithm is proved its performance in theoretically and practically in experimental result. The result shows the accuracy by using the LDA feature selection.All the limitations of existing image fusion methods including color distortion for the above said reason gives motivation for development of new improved image fusion method which will compensate these limitations. Thus our proposed algorithm is proved.

\section{References}

[1] Zhijun Wang, Djemel Ziou, Costas Armenakis, Deren $\mathrm{Li}$ and Qingquan Li, - AComparative Analysis of Image Fusion Methods", IEEE Transactions on Geoscience And Remote Sensing, Vol. 43, No. 6, June 2005, pp. $1391-1402$

[2] Veeraraghavan Vijayaraj, Nicolas H. Younan and Charles G. O'Hara, Concepts of Image Fusion in Remote Sensing Applications", IEEE, Geoscience and Remote Sensing International Symposium - IGARSS, 2006, pp. $3781-3784$

[3] Myungjin Choi, -A New Intensity-Hue-Saturation Fusion Approach to Image Fusion With a Tradeoff Parameter", IEEE Transactions on Geoscience And Remote Sensing, Vol. 44, No. 6, June 2006, pp. 1672 1682

[4] Shutao Li, Haitao Yin and Leyuan Fang, -Renote Sensing Image Fusion via Sparse Representations Over Learned Dictionaries", IEEE Transactions on Geoscience And Remote Sensing, Vol. 51, No. 9, September 2013, pp. 4779 - 4789

[5] Wenkao Yang, Jing Wang and Jing Guo, -ANovel Algorithm for Satellite Images Fusion Based on Compressed Sensing and PCA", Hindawi Publishing Corporation, Mathematical Problems in Engineering, Volume 2013, pp. 1 - 10

[6] Saad M. Darwish, Multi-level fuzzy contourlet-based image fusion for medical applications", IET Image Processing, Volume 7, Issue 7, ISSN 1751-9659, 2013, pp. $694-700$
[7] Chun-Liang Chien and Wen-Hsiang Tsai, Image Fusion With No Gamut Problem by Improved Nonlinear IHS Transforms for Remote Sensing”, IEEE Transactions on Geoscience And Remote Sensing, Vol. 52, No. 1, January 2014, pp. 651 - 663

[8] Heng Chu and Weile Zhu, Fusion of IKONOS Satellite Imagery Using IHS Transform and Local Variation", IEEE Geoscience And Remote Sensing Letters, Vol. 5, No. 4, October 2008, pp. 653 - 657

[9] Myungjin Choi, -A New Intensity-Hue-Saturation Fusion Approach to Image Fusion With a Tradeoff Parameter", IEEE Transactions on Geoscience And Remote Sensing, Vol. 44, No. 6, June 2006, pp. 1672 1682

[10] Frosti Palsson, Johannes R. Sveinsson, Magnus Orn Ulfarsson and Jon Atli Benediktsson, Model-Based Fusion of Multi- and Hyperspectral Images Using PCA and Wavelets", IEEE Transactions on Geoscience and Remote Sensing, Vol. 53, No. 5, May 2015, pp. 2652 2663.

[11] Shutao. Li, Bin. Yang," Multi focus image fusion based on wavelet and curvelet transform", Pattern recognition letters, 29:1295-1301, February 2008.

[12] Y. Zhang, Understanding image fusion," Photogramm. Eng. Remote Sens., vol. 70, no. 6, pp. 657-661, Jun. 2004.

[13] Krista Amolins, Yun Zhang, and Peter Dare, Wavelet based image fusion techniques-An introduction, review and comparison", ISPRSJournal of Photogrammetric and Remote Sensing, Vol. 62, pp. 249-263, 2007.

[14] Patil, Ujwala, and Uma Mudengudi. "Image fusion using hierarchical PCA." In image Information Processing (ICIIP), 2011 InternationalConference on, pp. 1-6. IEEE, 2011.

[15] Pei, Yijian, Huayu Zhou, Jiang Yu, and Guanghui Cai. "The improved wavelet transforms based image fusion algorithm and the qualityassessment." In Image and Signal Processing (CISP), 2010 3rd International Congress on, vol. 1, pp. 219-223. IEEE, 2010.

[16] Sruthy, S., Latha Parameswaran, and Ajeesh P. Sasi. "Image Fusion Technique using DT-CWT", IEEE International Multi-Conference on automation, computing,control, communication \& compressed sensing (iMac4S)", Kottayam, pp. 160-164, 22-23 March, 2013. 